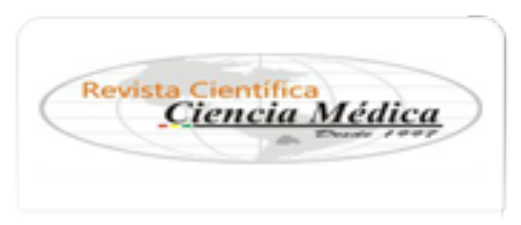

Revista Científica Ciencia Médica

ISSN: 1817-7433

ISSN: 2220-2234

revista_cienciamedica@hotmail.com

Universidad Mayor de San Simón

Bolivia

Anaya Muño, Santiago Franklin; Calvo Orellana, Eldy Ericka; Valdez

Ramallo, María Alexandra; Santa Cruz Rodriguez, Adriana C.

ACTIVIDAD ANTIMICROBIANA DE WIRA WIRA Y CERRAJA CONTRA

ESTAFILOCOCO, ENTEROCOCO, PSEUDOMONAS Y ESCHERICHIA

Revista Científica Ciencia Médica, vol. 23, núm. 1, 2020, -Junio, pp. 15-21

Universidad Mayor de San Simón

Cochabamba, Bolivia

Disponible en: http://www.redalyc.org/articulo.oa?id=426064021002

Cómo citar el artículo

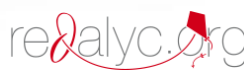

Número completo

Más información del artículo

Página de la revista en redalyc.org

Sistema de Información Científica Redalyc Red de Revistas Científicas de América Latina y el Caribe, España y Portugal Proyecto académico sin fines de lucro, desarrollado bajo la iniciativa de acceso abierto 


\title{
ORIGINAL
}

\section{ACTIVIDAD ANTIMICROBIANA DE WIRA WIRA Y CERRAJA CONTRA ESTAFILOCOCO, ENTEROCOCO, PSEUDOMONAS Y ESCHERICHIA}

\author{
ANTIMICROBIAL ACTIVITY OF WIRA WIRA AND CERRAJA AGAINST \\ STAPHYLOCOCCUS, ENTEROCOCCUS, PSEUDOMONAS AND \\ ESCHERICHIA.
}

\begin{abstract}
Santiago Franklin Anaya Muñoz', Eldy Ericka Calvo Orellana', María Alexandra Valdez Ramallo', Adriana C. Santa Cruz Rodriguez ${ }^{2}$
\end{abstract}

\section{RESUMEN}

Introducción: El uso de plantas con diversos fines etnomedicinales es una práctica ancestral y actualmente común en una vasta parte de la población de Bolivia.

Objetivo general: Identificar si la wira wira y cerraja de nuestro medio poseen actividad antimicrobiana contra bacterias patógenas y determinar su toxicidad a través del bioensayo de pruebas biológicas.

Materiales y Métodos: Estudio descriptivo; Universo, Wira Wira recolectada del Valle Alto de Cochabamba y Cerraja de Cercado; Muestra, se obtuvo de forma aleatoria un kilogramo deWira Wira y Cerraja respectivamente; Métodos, se emplearon diferentes técnicas para la extracción de los principios activos, se realizaron pruebas biológicas para determinar la toxicidad de los mismos y se determinó la actividad antimicrobiana por el método de difusión en agar.

Resultados: Wira wira demostró actividad antimicrobiana con 4 extractos; alcohólico de tallo, flor y alcohólico agotado de hojas frente a E. faecalis y S. aureus, y acuoso de hojas frente a P. aeruginosa y S. aureus. Por otro lado no se evidencio actividad antibacteriana de los extractos de cerraja.

Conclusiones: Wira wira es candidata para estudios posteriores destinados a la identificación del o los compuestos activos puros con actividad antibacteriana, hecho respaldado por los resultados obtenidos en esta y otras investigaciones.

Los extractos de cerraja, a diferencia de los resultados obtenidos de otros trabajos, no presentaron actividad antimicrobiana, demostrando la posibilidad y vinculación de la variación en los compuestos de la planta con el ecosistema en el que se desarrolla.

\section{ABSTRACT}

Introduction: The use of plants with various ethnomedicinal purposes is an ancestral practice and currently common in a vast part of the population of Bolivia.

General objective: To identify if the extracts of both plants present in our environment have antimicrobial activity against pathogenic bacteria and to determine their toxicity through the bioassay of biological tests.

Materials and Methods: Descriptive study; Universe, Wira Wira collected from Valle Alto in Cochabamba and Cerraja collected from Cercado; Sample, we obtained one kilogram of each, Wira Wira and Cerraja randomly; Methods, different techniques were used to extract the active ingredients, biological tests were performed to determine their toxicity and antimicrobial activity was determined by the agar diffusion method.

Results: Wira Wira demonstrated antimicrobial activity with 4 extracts; stem alcoholic, flower and alcoholic depleted of leaves against E. faecalis and S. aureus, and aqueous of leaves against P. aeruginosa and S. aureus. On the other hand, there was no evidence of antibacterial activity with the cerraja extracts.

Conclusions: Wira Wira is a candidate for further studies aimed at the identification of the pure active compound (s) with antibacterial activity, all this backed up by the positive results in the agar diffusion tests grown with pathogenic bacteria.

Unlike the results that demonstrate the antibacterial activity of the Cerraja in research carried out in other parts of the world, it is not the case of the one in our territory, whose extracts did not present any antimicrobial activity, demonstrating the possibility and linking the variation in plant compounds with the ecosystems in which it develops.
1 Estudiante de Medicina de la Universidad Mayor de San Simón, Facultad de Medicina Aurelio Meleán CochabambaBolivia.

2 Master en Microbiología, Docente de la Universidad Mayor de San Simón, Facultad de Medicina Aurelio Meleán Cochabamba-Bolivia.

Correspondencia a:

Santiago Franklin Anaya Muñoz

Correo: Santiagoanaya98@hotmail.com

Teléfono: +59176984255

Palabras clave: Técnicas In Vitro, Agentes Antibacterianos, Achyrocline, Sonchus.

Keywords: In Vitro Techniques, Anti-Bacterial Agents, Achyrocline, Sonchus.

Procedencia y arbitraje: no comisionado, sometido a arbitraje externo.

Recibido para publicación: 22 de abril de 2019 Aceptado para publicación: 11 de abril de 2020

Citar como:

Anaya Muñoz SF, Calvo Orellana EE, Valdez Ramallo MA, Santa Cruz Rodriguez AC. Actividad antimicrobiana de achyrocline satureioides y sonchus oleraceus contra staphylococcus aureus, enterococcus faecalis, pseudomona aeuroginosa y escherichia coli. Rev Cient Cienc Med 2020;23(1): 15-21 


\section{INTRODUCCIÓN}

Dolivia es un país que cuenta con una magna riqueza natural, destacando la amplia variedad en la flora. Diversas especies de plantas son utilizadas en la práctica de la medicina tradicional y empírica por sus efectos simbólicos o reales para el ser humano, sobre todo en las poblaciones rurales ${ }^{1}$. Achyrocline satureioides (wira wira) especie perteneciente a la familia de las asteráceas, es una planta medicinal utilizada ampliamente en muchas regiones de los Andes de Bolivia por sus propiedades antiinflamatorias, antiespasmódica, antitusígena, así como el empleo sobre heridas superficiales para evitar infecciones ${ }^{2-5}$. Su conocida actividad antimicrobiana se relaciona a la presencia de metabolitos secundarios, como ser: flavonoides, esteroles, triterpenos, saponinas, ácidos cafeoilquínicos y aceites esenciales, que otorgan esta cualidad a la planta ${ }^{6,7}$. Sonchus oleraceus (cerraja) es una planta nativa de Europa, Mediterráneo y el occidente de Asia. Ha sido introducida en el resto del mundo y se reporta estudios en los cuales se encuentra actividad antimicrobiana atribuida a los compuestos fenólicos (flavonoides y cumarinas) que contiene esta planta. También son conocidas las propiedades etnomedicinales, las infusiones son empleadas para tratar los síntomas del resfrío, dolores de garganta, diarrea, y heridas infectadas ${ }^{8-12}$.El presente trabajo se realiza con el fin de determinar el poder antibacteriano que poseen estas dos plantas en nuestra región (Cercado y Valle Alto de Cochabamba), en vista de que puede existir variaciones en los compuestos activos de las plantas de acuerdo a la zona geográfica en la cual se desarrollan, pudiendo así ocasionar diferencias en la actividad que tienen contra las bacterias patógenas.

\section{MATERIALES Y MÉTODOS}

Se realizó un estudio de tipo descriptivo donde las muestras seleccionadas fueron 2 géneros de plantas medicinales pertenecientes a la misma familia; Achyrocline satureioides (wira wira) obtenida del Valle Alto de Cochabamba y Sonchus oleraceus (cerraja) obtenida de Cercado - Cochabamba y 4 tipos de bacterias; Staphylococcus aureus 25923, Enterococcus faecalis
29212, Pseudomonas aeuroginosa 27853, y Escherichia coli 25922 (cepas ATCC).

Las variables independientes fueron las plantas de estudio de las cuales se obtuvieron extractos acuosos por hidrodestilación, extractos alcohólicos y alcohólico agotado de raíz, hoja, tallo y flor de Achyrocline satureioides y Sonchus oleraceus. (Figura 1)

Para realizar el Bioensayo se obtuvieron quistes de artemias, los cuales fueron incubados en las condiciones necesarias para conseguir su eclosión y desarrollo. Posteriormente se expusieron grupos de 10 artemias adultas a diferentes concentraciones de los extractos (10, $20,50,70$ y $100 \mathrm{mg} / \mathrm{ml}$ ) en viales con $5 \mathrm{ml}$ de solución salina durante 24 horas.

Las variables dependientes, sensibilidad y resistencia, se determinaron a través del método de difusión en agar, para lo cual se fabricaron sensidiscos de $6 \mathrm{~mm}$ de diámetro por 0.5 de ancho, impregnados a diferentes concentraciones de los extractos $(3,125 ; 6,25$; 12.5; 25; 50; $100 \mathrm{mg} / \mathrm{ml}$ ). Los mismos fueron aplicados en la superficie de un cultivo inoculado con bacterias. El antimicrobiano difundió desde el disco al medio de cultivo produciendo una zona de inhibición las cuales fueron medidas respecto al diámetro ocasionado.

La investigación tuvo lugar en el centro de tecnología agroindustrial del laboratorio de pruebas biológicas de la Universidad Mayor de San Simón, realizándose en sus instalaciones el Bioensayo con artemias y el método de difusión en agar. Con los datos recolectados se realizó el estudio que consistió en organizar, relacionar y analizar la información utilizando los programas Excel y Word. No se manipularon resultados en el presente trabajo.

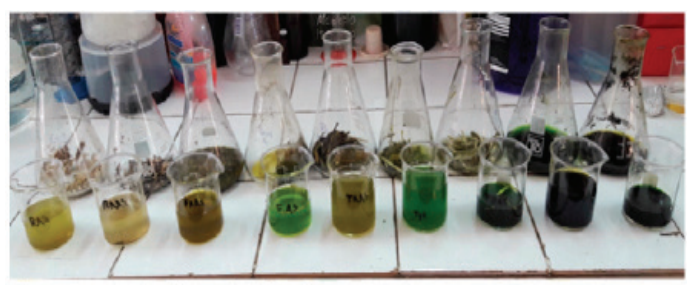

Figura 1. Extractos alcohólicos de sonchus aleroceus

\section{RESULTADOS}

A través del bioensayo de pruebas biológicas se determinó la toxicidad y mortalidad que causaron los diferentes extractos de estas plantas frente a la artemia salina. Se 
Tabla 1. Bioensayo con artemias - toxicidad de los extractos de wira wira.

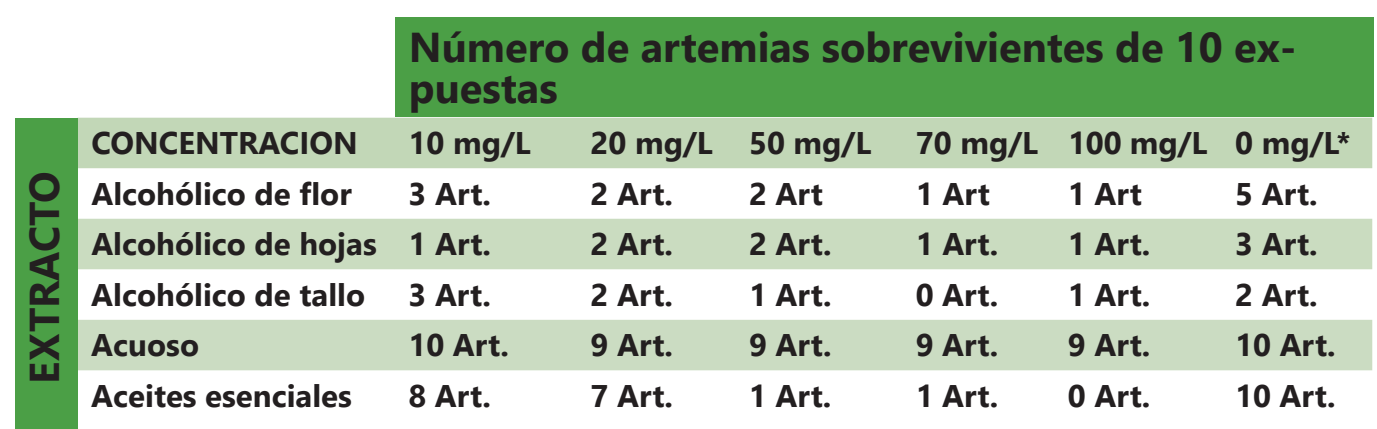

Tabla 2. Bioensayo con artemias - toxicidad de los extractos de cerraja.

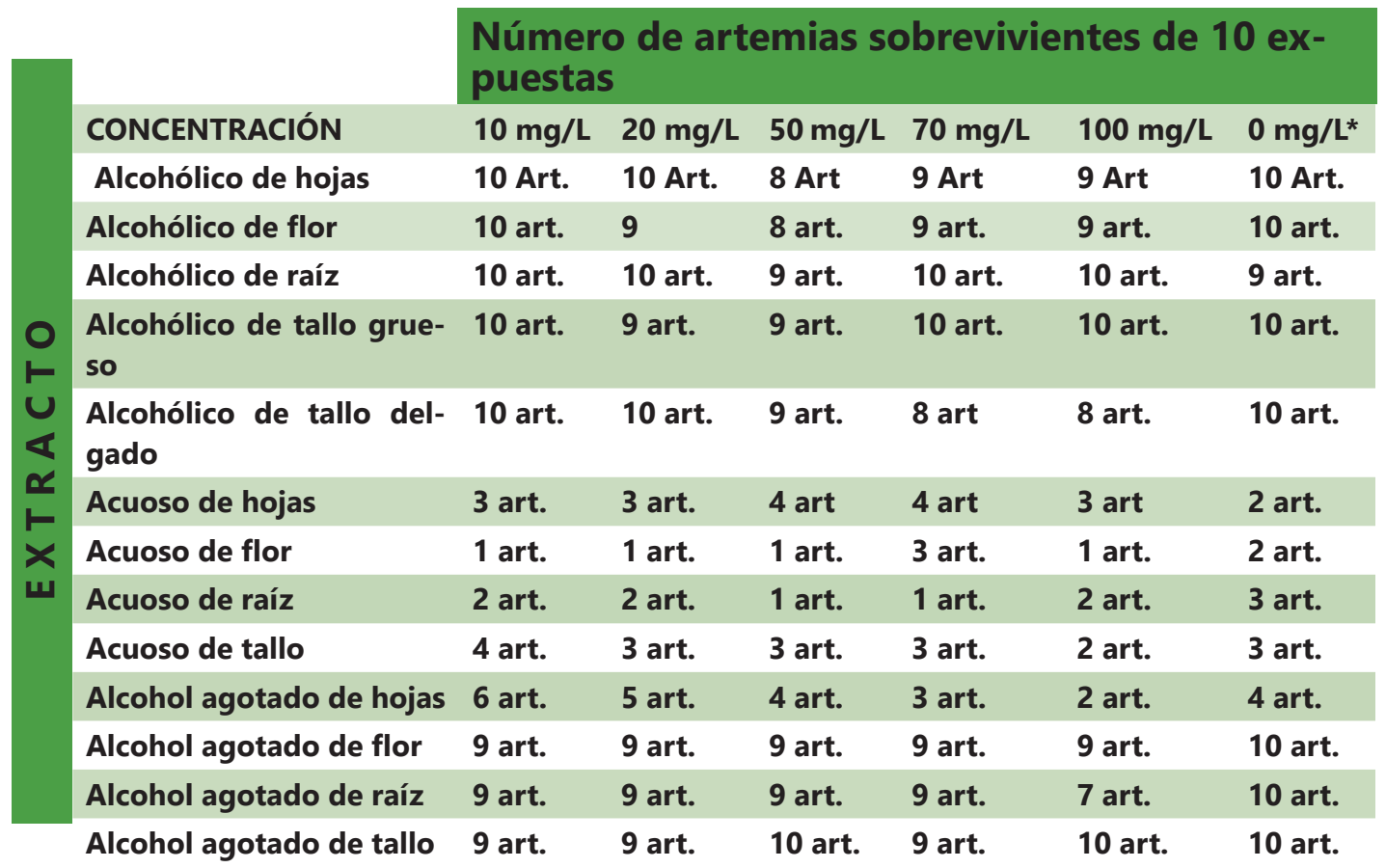

*Grupo de artemias testigo no expuestas a los extractos

Nota. Revisar la sección de discusión en la cual se aclara la posible causa de la muerte de las artemias en el grupo testigo.

encontraron altos niveles de mortalidad en los grupos de artemias expuestas a los componentes de Wira Wira, entre un $65 \%$ a $95 \%$ perecieron al entrar en contacto durante 24 horas con las diferentes concentraciones de los extractos (Tabla 1). De la cerraja se obtuvieron resultados que no sobrepasaron el $20 \%$ de mortalidad frente a los extractos alcohólicos a concentraciones de $100 \mathrm{mg} / \mathrm{ml}$. Sin embargo, las artemias expuestas a los extractos acuosos presentaron una mortalidad de 65 a $90 \%$ a concentraciones de $10 \mathrm{mg} / \mathrm{ml}$. (Tabla 2). En los ensayos de susceptibilidad antimicrobiana por difusión en agar frente a $S$. aureus y $\mathcal{E}$. faecalis se observó que el extracto alcohólico de tallo fue el que presentó mayor efectividad con un halo de inhibición de $9 \mathrm{~mm}$ de diámetro a la concentración mínima inhibitoria de 3.125 $\mathrm{mg} / \mathrm{ml}$. En el caso del extracto alcohólico de flor este demostró un halo de $9-11 \mathrm{~mm}$ frente a estas bacterias a concentraciones de $6.25 \mathrm{mg} / \mathrm{ml}$. Ambos extractos causaron halos inhibitorios con un rango de 18 a $24 \mathrm{~mm}$ de diámetro a concentraciones de 50 y $100 \mathrm{mg} / \mathrm{ml}$ respectivamente. El extracto acuoso de hojas fue el único que inhibió a $\mathscr{P}$. aeruginosa con un halo de $8 \mathrm{~mm}$ de diámetro a una concentración de $100 \mathrm{mg} / \mathrm{ml}$. (Tabla 3). No se demostró ningún halo de inhibición en los cultivos expuestos a los extractos de la Cerraja. 
Tabla 3. Actividad antimicrobiana de wira wira

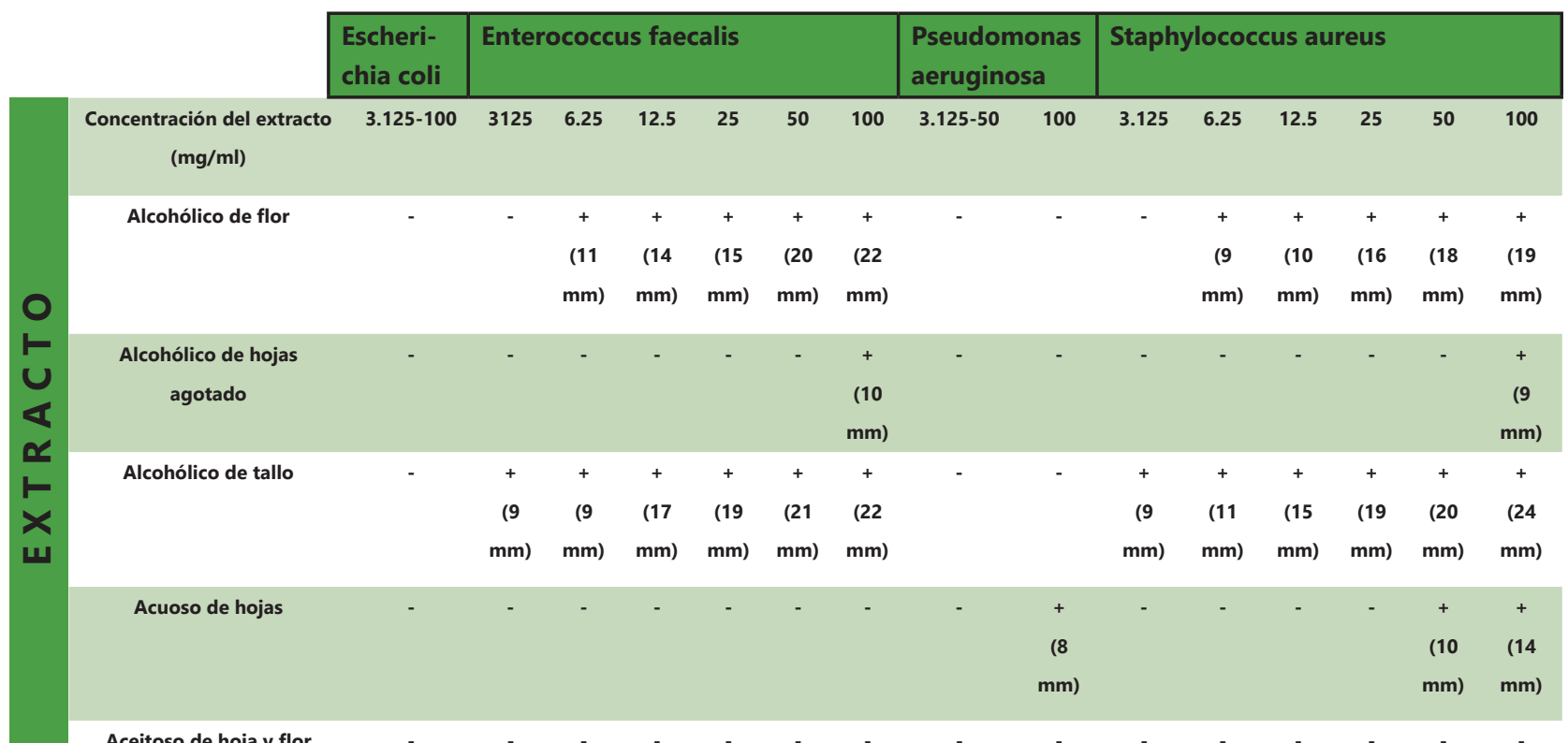

\section{DISCUSIÓN}

La actividad antimicrobiana que se obtiene con los extractos del tallo y flor de wira wira frente a $S$. aureus y $\mathcal{E}$. faecalis son comparables a los demostrados en otras investigaciones donde se utiliza partes iguales de la planta y las mismas cepas ATCC. ${ }^{2-4,18,19}$ (Figura 2). Por otro lado, se encuentra actividad antimicrobiana en el extracto acuoso de hojas de wira wira contra P. aeruginosa, hecho que destaca al ser esta una bacteria fármaco resistente y estar relacionada a muchas patologías ${ }^{20-22}$. E. coli demuestra resistencia a los extractos de wira wira en esta investigación al igual que en otras.

Nuestros resultados sugieren que existe una actividad antibacteriana más efectiva frente a bacterias grampositivas, lo cual puede obedecer a que las gramnegativas poseen una membrana externa y una espacio periplásmico único. (Duffy and Power, 2001) ${ }^{23}$.

No se obtuvo resultados que evidencien poder antimicrobiano de los extractos obtenidos de cerraja procedente de Cercado - Cochabamba frente a las cepas problema, hallazgo que difiere de otras investigaciones en las que se demuestra la actividad antimicrobiana ${ }^{8,11,12}$. Esta diferencia puede deberse a los múltiples factores que afectan la composición de la planta, desde el ecosistema en el que se desarrolla hasta los factores ambientales de cada región ${ }^{24}$.

Debe considerarse que en esta investigación se trabajó con extractos no purificados de ambas plantas lo cual reduce en gran medida la actividad antibacteriana real de las sustancias puras que poseen las mismas, requiriendo así dosis de mayor concentración para producir halos de inhibición bacteriana.

El bioensayo con artemias se realiza con el fin de detectar la bioactividad de los compuestos heterogéneos de los extractos de wira wira y cerraja sin identificar ni purificar un compuesto activo específico, permitiendo así determinar el efecto letal de los mismos en organismos vivos ${ }^{13-15}$. Resulta importante su aplicación

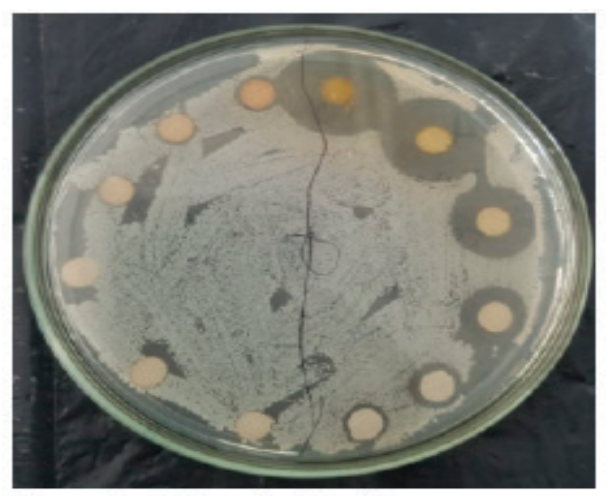

Figura 2. Cultivo de Staphylococcus aureus expuesto a extracto alcohólico de tallo en Wira Wira (lado derecho de la imágen) y extracto alcohólico de hojas agotado de Wira Wira (lado izquierdo) 
en el descubrimiento y desarrollo de nuevas sustancias de origen natural con un potencial favorable a poseer actividad antimicrobiana y un posible paso para el desarrollo de nuevos fármacos. Los extractos de wira wira que presentan mayor actividad antimicrobiana causan una elevada mortalidad en los grupos de artemias. Se observa que las artemias testigo, grupo no expuesto a la intervención, perecen en proporción comparable a las que son expuestas a los extractos, hecho que no se evidencia en los demás grupos. Esto podría ocurrir por una propiedad de alta volatilización de un compuesto de la planta que contaminaría los medios viales de las artemias testigo, o bien por las variantes del desarrollo de las artemias según Vanhaecke y Persoone ${ }^{15,16}$.

En el caso de la cerraja, los extractos acuosos y extractos alcohólicos agotados de hojas presentan un nivel de toxicidad considerable en los grupos de artemias expuestas respectivamente, incluyendo a los grupos testigo. Se obtienen bajos niveles de mortalidad con los extractos restantes.

Con la presente investigación podemos concluir que en Cochabamba - Bolivia, Achyrocline satureioides posee actividad antibacteriana, resaltando la obtenida de los extractos de flor y tallo. Por otro lado no se evidencia poder antimicrobiano de los extractos que se obtienen de Sonchus oleraceus frente a las cepas problema. Los extractos de Achyrocline satureioides demuestran altos niveles de mortalidad en el bioensayo con artemias, hecho que no se observa con la mayoría de los extractos de Sonchus oleraceus.

\section{AGRADECIMIENTOS}

Al Centro de Tecnología Agroindustrial del laboratorio de pruebas biológicas de la Universidad Mayor de San Simón por facilitar sus instalaciones para que fuera posible la realización de esta investigación. Al Lic. M. Sc. Ricardo Sahonero Irahola por el conocimiento brindado facilitando recursos para el desarrollo de este trabajo. A la Magister Adriana Santa Cruz por su gran apoyo, asesoría y gran interés en ayudarnos a alcanzar esta meta. A nuestros colegas de investigación en especial a Horacio Villaroel Villazón y Paul Cavero Del Granado por su participar en la recolección de datos para la realización de este proyecto. 
1. De la Riva V, Jai P. Plantas medicinales en los Andes de Bolivia. En: Moraes M., Øllgard B, Lars P, Borchsenius $\mathrm{F}$, Balslev $\mathrm{H}$, editores.Botánica Económica De Los Andes Centrales. Ed. La Paz, Bolivia - Universidad Mayor de San Andrés : 2006. p. 268-84.

2. Choque C, Mariza M, Pauro Q, Elvira V. Evaluación de la actividad antimicrobiana in vitro del extracto de Achyrocline alata (huira huira) en cepas de Staphylococcus aureus, Escherichia coli y Pseudomonas aeruginosa [dissertation]. Arequipa (AR): Universidad Católica de Santa María; 2017.

3. Casero C, Machín F, Méndez-Álvarez S, Demo $M$, Ravelo ÁG, Pérez-Hernández N. Structure and Antimicrobial Activity of Phloroglucinol Derivatives from Achyrocline satureioides. PubMed [Internet].2015[Citado en abril 2019];23;78(1):93102. Disponible en: https://www.ncbi.nlm.nih.gov/ pubmed/25517209

4. Corrêa JM,Marchionatti CA, Spaniol B, Petrovick PR. Atividade desinfetante anti-Staphylococcus aureus meticilina resistentes e compostos flavonóides em Achyrocline satureioides Lam. (macela). Rev Cubana Plant Med [Internet]. 2016 [Citado en abril 2019];21(4). Disponible en: http://revplantasmedicinales.sld.cu/ index.php/pla/article/view/515/205

5. Arrázola Rivero S, Atahuachil M, Saravia E, Lopez A. Diversidad florística medicinal y potencial etnofarmacológico de las plantas de los valles secos de cochabamba - Bolivia. Revista Boliviana de Ecología y Conservación Ambiental. 2002 [Citado en abril 2019];12:53-85.33p.Disponible en: https:// www.researchgate.net/profile/Margoth_Atahuachi/ publication/292405139_Diversidad_floristica_ medicinal_y_potencial_etnofarmacologico_de_las_ plantas_de_los_Valles_Secos_de_Cochabamba/ links/58cb26f092851c31f6552369/Diversidadfloristica-medicinal-y-potencial-etnofarmacologicode-las-plantas-de-los-Valles-Secos-de-Cochabamba. pdf

6. Gómez M, Vásquez N. Evaluación de la susceptibilidad de campylobacter jejuni frente a las especies: Achyrocline satureioides, Achyrocline bogotensis y Gnaphalium elegans [dissertation]. Bogotá (BOG): UNIVERSIDADDECIENCIAS APLICADAS Y AMBIENTALES U.D.C.A; 2018. Disponible en: https:// repository.udca.edu.co/handle/11158/1353

7. Vega J. Contribución a la estandarización del proceso de obtención de un extracto a escala de laboratorio a partir de las partes aéreas de Achyrocline satureioides (Lam.) D.C [dissertation]. Bogotá (BOG): Universidad Nacional de Colombia Facultad de Ciencias Departamento de Farmacia;
2016. Disponible en: https://repositorio.unal.edu.co/ handle/unal/64072

8. Sulca TS. Determinación de la actividad antimicrobiana de los extractos de Acmella repens (Botoncillo) Urtica dioca (Ortiga Negra) y Sonchus oleraceus (Kana yuyo) plantas registradas en la parroquia La Esperanza - Imbabura, sobre Staphylococcus aureus, Pseudomonas aeruginosa, y Candida albicans, causantes de enfermedades bucofaríngeas [dissertation]. Sangolquí (QUI): Escuela politécnica del ejército; 2010.

9. Department of Pharmacognosy, Faculty of Pharmacy, Al-Azhar University. Cytotoxic and Antibacterial Constituents from the Roots of Sonchus oleraceus L. Growing in Egypt. PHCOG MAG [Internet] 2009 [Citado en febrero 2020]; 5:324-28. Disponible en: http://www.phcog.com/article.asp?issn=09731296; year $=2009 ;$ volume $=5$;issue =20; spage=324; epa ge $=328$; aulast $=$ Elkhayat

10. M. T. Pretel, M. Sánchez, V. Pérez, C. Obón. Usos y propiedades de las plantas comestibles silvestres de la familia Asteráceas. Horticultura [Internet] 2008 [Citado es febrero 2020]; 207: 46-53. Disponible en: http://www.horticom.com/revistasonline/ horticultura/rh207/46_53.pdf

11. Li W, Sun H, Zhou J, Zhang Y, Liu L, Gao Y. Antibacterial activities, anti-oxidant contents and anti-oxidant properties of three traditional Chinese medicinal extracts. Bangladesh Journal of Pharmacology [Internet].2015 [Citado en Marzo 2019];10: 131-37.9. Disponible en: https://www. banglajol.info/index.php/BJP/article/view/21324

12. Mejía EM, Aredo AC, Argomedo CA, Araujo WS, Aranda JM. Efecto antibacteriano in vitro entre Sonchus oleraceus y Ceftazidima contra Pseudomonas aeruginosa. Rev méd Trujillo [Internet].2018 [Citado en arbil 2019];13(3):12230. Disponible en: https://www.researchgate.net/ publication/328823001_Efecto_antibacteriano_in_ vitro_entre_Sonchus_oleraceus_y_Ceftazidima_ contra_Pseudomonas_aeruginosa

13. Aida $M$, Vinueza $D$, Acosta $K$, Toaquiza C, Mendez E, Arias F. Estudio fitoquímico preliminar y evaluación de la actividad citotóxica del látex de Euphorbia laurifolia Juss. ex. Lam sobre Artemia salina. Revistas EPOCH [Internet]. 2018 [Citado en abril 2019];2(20).8p. Disponible en: http://dspace. espoch.edu.ec/handle/123456789/9383

14. Saetama V, Vera L, Vanegas M.E, Cruzat C, Brazales D. Evaluación toxicológica de soluciones acuosas de ibuprofeno mediante bioensayos con Artemia salina, Allium schoenoprasum L y Lactuca 
sativa. AETOX [Internet]. 2018 [Citado en abril 2019]; 35:112-118.7p. Disponible en: http://rev.aetox. es/wp/wp-content/uploads/2018/12/Revista-deToxicologia-35.2-34-40.pdf

15. Pérez OP, Lazo F. Ensayo de artemia: útil herramienta de trabajo para ecotoxicólogos y químicos de productos naturales. Revista de protección vegetal [Internet]. 2010 [Citado 2019 Marzo 22];v.25.10p. Disponible en: http://scielo.sld.cu/scielo.php?script=sci_ arttext\&pid=S1010-27522010000100008

16. Vanhaecke P, Persoone G. The ARC-Test: a standardized short-term routine toxicity test with Artemia nauplii. Methodology and evaluation. Ecotoxicological Testing for the Marine Environ [Internet]. 1984 [Citado en abril 2019] ;143-157. Disponible en: http://www.vliz.be/nl/open-marienarchief? module=ref\&refid=3275

17. Bernal MR, Guzmán, M. El Antibiograma de discos - Normalización de la técnica de Kirby-bauer. Biomédica [Internert]. 1984 [Citado en Marzo 2019] 4(3-4), 112-121. 10p. Disponible en: https://www. revistabiomedica.org/index.php/biomedica/article/ view/1891

18. Cruz AT. Acción Antimicrobiana del Extracto Etanolico del Gnaphalium Vira Vira (Wira Wira) [dissertation]. Puno : Universidad Nacional del Altiplano Puno - Escuela de Post Grado - Maestria en Salud Pública; 2014. 81p.

19. Correa JM. Atividade antibacteriana de desinfetantes convencionais e de extrações de Achyrocline satureioides (Lam.) DC. (Asteraceae) ("macela") sobre Staphylococcus aureus meticilina resistentes (MRSA) [dissertation]. Porto Alegre : Universidade Federal do Rio Grande do Sul. 2013. $103 p$.

20. MurrayPR, Rosental KS, PfallerMA. Microbiologia medica. 8a ed. Barcelona (ES): Elsierver; 2017.

21. Tapia JE, Gomez A, Zeballos JM, Crespo C, Santa Cruz A. Identificación y Antibiograma de Pseudomonas aeruginosayStaphylococcusaureus en el Pabellón Infantil de Quemados del Hospital Viedma Agosto-2013. Rev Cient Cienc Méd [Internet].2015 [Citado en abril 2019];v.17n.1. Disponible en: http://www.scielo.org.bo/scielo.php?script=sci_ arttext\&pid=S1817-74332014000100006

22. Weinstein M, Patel JB, Campeau S, EliopoulosGM, Galas MF,Humphries RM, et al. Performance Standards for Antimicrobial Susceptibility Testing [Internet]. 28th Edition. Pensilvania (USA): Clincal and Laboratory Standars Institue; 2018 [Citado en Marzo 2019]. Disponible en: http://file.qums.ac.ir/ repository/mmrc/CLSI-2018-M100-S28.pdf

23. Duffy CF, Power RF. Antioxidant and antimicrobial properties of some Chinese plant extracts. International Journal of Antimicrobial Agents [Internet].2001 [Citado en marzo 2019];(6):527.3p. Disponible en: https://www.ncbi.nlm.nih.gov/ pubmed/11434342

24. Guariguata MR, Kattan GH. Ecología y conservación de bosques neotropicales. Primera Edición. Cartago (CR): Editorial Tecnológica : Libro Universitario Regiona; 2002. 